\title{
Proteomics as a Tool to Decipher Novel Mechanistic Candidates in Cardiac Pathophysiology
}

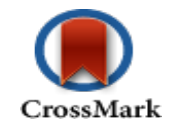

\author{
Kaberi Datta, Sagartirtha Sarkar*
}

Genetics and Molecular Cardiology Laboratory, Department of Zoology, University of Calcutta, Ballygunge Circular Road, Kolkata, West Bengal, India

*Corresponding author: Sagartirtha Sarkar, Genetics and Molecular Cardiology Laboratory, Department of Zoology, University of Calcutta, 35, Ballygunge Circular Road, Kolkata-700019, West Bengal, India, Tel: 0091-33-2461-5445; Fax: 0091-33-2241-3222; E-mail: sagartirtha.sarkar@gmail.com

\begin{abstract}
Cardiac diseases resulting in heart failure have become one of the major causes of mortality as well as reduced quality of life for people all over the world. In an effort to understand the mechanisms underlying cardiac diseases and devise preventive as well as therapeutic measures, a lot of research has been carried out over the years. Two systems biology approaches- proteomics and computational modelling have drastically changed the field of cardiac research by delivering vast amounts of relevant molecular data thus enhancing the interpretation of both clinical as well as subcellular events dictating pathophysiology during compromised heart function. This review specifically focuses on the contributions of such systems biology approaches in proper understanding of the pathophysiology of diseased myocardium.
\end{abstract}

Received Date: August 04, 2015

Accepted Date: November 12, 2015

Published Date: November 17, 2015

Citation: Sarkar, S., et al. Proteomics as a Tool to Decipher Novel Mechanistic Candidates in Cardiac Pathophysiology. (2015) Bioinfo Proteom Img Anal 1(2): 51- 55.

DOI: $10.15436 / 2381-0793.15 .013$

Keywords: Proteomics; Computational modelling; Heart failure; Cardiac diseases

\section{Introduction}

Cardiovascular diseases resulting in heart failure remain one of the leading causes of mortality the world over. They were accountable for $31 \%$ of global deaths in $2012^{[1]}$. In view of this, a huge amount of research has been targeted towards diagnoses, prevention and therapeutic intervention of cardiovascular disease forms with a mind to abate the incidence of heart failure. While experimental research has given the bulk of breakthroughs, management procedures and understanding of cardiovascular diseases, a systems biology approach is enhancing and reshaping the way research is carried out and data is interpreted in this field. It is yielding such beneficial results of itself and in conjunction to experimental biology that would not be possible for the latter alone. The purpose of this review is to succinctly catalogue two relevant systems biology approaches being used currently in cardiac biology and highlighting the important contributions made by them.

\section{Proteomics in cardiac biology}

Proteome is the entire complement of proteins along with their modifications produced by a cell or organism that varies with time and physico-chemical set up of the cell or tissue. Proteomics is a term that applies to large scale and comprehensive study of any specific proteome in order to understand cellular processes. Proteomics approaches have helped in a wide array of cardiac research like biomarker discovery for different cardiac diseases to aid in efficient diagnoses and treatment, cellular signalling networks involved in the pathology of different cardiac diseases, understanding the cellular basis of disease initiation and progression and action of therapeutic drugs in different cardiac diseases.

While expression proteomics have yielded a large amount of information about the proteins involved in any cardiac disease, structural proteomics is giving specific information regarding protein complexes ${ }^{[2]}$, their functions, drug- protein interactions as well as drug-off target protein interactions ${ }^{[3,4]}$, to enhance therapeutic approaches. Different protein separation techniques are 
used prior to mass spectrometry to enhance the resolution power of the mass spectrometer and enable it to detect rare proteins. 2 dimensional difference in gel electrophoresis (DIGE), isobaric tags for relative and absolute quantitation (iTRAQ ${ }^{\mathrm{TM}}$ ) labelling and liquid chromatography (LC) are some popularly used labelled separation techniques that allow multiplexing of samples for ease in comparison ${ }^{[5-9]}$.

\section{Proteomics in biomarker discovery}

Biomarkers are measurable indicators of cardiac disease presence and/ or severity. Novel candidates are always being researched which can be easily assessed noninvasively and provide high degree of specificity and sensitivity in diagnosing different forms of cardiac diseases. As of now the established biomarkers in clinical use are- cardiac Troponin I and $\mathrm{T}$ for diagnosing acute myocardial infarction (MI), B-type natriuretic peptides (BNP) for diagnosing heart failure, $\mathrm{C}$ reactive protein as inflammation marker of ischemic disease ${ }^{[10-12]}$. Towards this end, proteomics has been an invaluable tool that has churned out several promising novel biomarker candidates. Cardiac myosin-binding protein $\mathrm{C}$ has been found to be a potential biomarker for early myocardial injury during acute $\mathrm{MI}^{[13]}$. Haptoglobin has also been found as a potential marker for acute $\mathrm{MI}^{[14]}$. A plasma proteomics approach has revealed Quiescin Q6 (QSOX1), a protein involved in forming disulfide bridges to be a novel biomarker for acute decompensated heart failure. When combined with existing BNP it became an even more specific and accurate diagnostic tool for acute decompensated heart failure ${ }^{[15]}$.

A subsection of patients with heart failure are often presented with preserved ejection fraction (HFEF) and as such their detection and diagnosis becomes difficult without the significant helping aid of echocardiographic parameters. A proteomic study with serum samples of patients with HFEF has found Angiogenin to be a promising biomarker for the condition along with a panel of 16 other proteins that could be studied further to develop one or more for clinical use ${ }^{[16]}$. Novel proteomic approach has been applied to find out protein differences in formalin fixed paraffin embedded samples of acute lethal myocardial infarctions that result in sudden death. This process can potentially solve the barrier of fresh tissue/ serum/ plasma samples for analysis. It is also one of the few studies that looked into the proteome of lethal myocardial infarction to find out that Sorbin and $\mathrm{SH} 3$ domain containing protein 2 (SORBS2) can serve as markers for very early phase of myocardial infarction with elevated serum concentrations ${ }^{[17]}$. There are other studies which are trying to address biomarker research for the benefit of cardiovascular therapy. As can be seen, proteomics provides a very useful and convenient tool for biomarker discovery that may be further developed for clinical use.

\section{Proteomics for elucidation of molecular processes in cardiac disease}

Proteomics has been used extensively for the understanding of molecular and cellular basis of different cardiac diseases. Most of proteomics research has been geared towards unravelling the pathophysiology of heart failure. Global proteomic differences between ischemic and non ischemic failing hearts, between ischemic and dilated cardiomyopathic heart failure, in early and end stage heart failure have been analysed ${ }^{[18-21]}$ while myocardial infarction has also been extensively studied ${ }^{[7,14,17]}$.
Myocardial infarction being a very critical factor for cardiac dysfunction or sudden death, has also received a high degree of proteomic research efforts. From the proteins specifically altered during MI and afterwards, to the discovery of biomarkers, to the difference it represents with other forms of cardiac disease such as hypertrophy, all have been explored by intensive proteomic tools ${ }^{[7]}$. Aside from the major share of studies elucidating these two cardiac dysfunctions, a fair number have also been dedicated to finding out the cellular protein mediated mechanisms behind atrial fibrillation, pressure overload hypertrophy, diabetic cardiomyopathy and others.

Besides global proteome changes in the above conditions, several studies have now targeted precise aspects of the protein machinery like the status of phosphorylation and oxidation on the one hand and sub cellular organelle protein milieu like that of mitochondria and myofilaments on the other to find out explicit information regarding all aspects of cardiac diseases.

In case of subcellular organelle proteomics, the advantage lies in the fact that greater amplification of a small section of cellular proteins leads to the identification of low abundance proteins which would otherwise be difficult ${ }^{[22]}$. Proteomic studies have indicated the involvement of mitochondrial pathways for TCA cycle either by the cycle enzymes or via $\mathrm{NADH}^{[23,24]}$, fatty acid oxidation and oxidative phosphorylation to be altered during various forms of cardiac failure ${ }^{[2]}$. Phosphoproteome analysis of the mitochondrial proteome provides further insights into the above mentioned processes and the post translational modifications (PTMs) of the concerned proteins ${ }^{[22]}$. One study has devised a proteomic approach that best covers the maximum repertoire of mitochondrial proteins ${ }^{[25]}$. They have found that SDS-PAGE followed by LC-MS/MS provided the best coverage of the mitochondrial proteome. Distinctions between mitochondrial proteome are also made based on the location- subsarcolemmal and inter fibrillar. Most work has been done with subsarcolemmal mitochondria. Mitochondrial phosphoproteome is being explored to acquire better understanding of the role of mitochondrial processes in heart failure ${ }^{[26,27]}$. One study using a rat model of human congestive heart failure has mapped 26 mitochondrial proteins which show phosphorylation in 39 sites characterised by 42 phosphopeptides ${ }^{[26]}$. Among the proteins changed by phosphorylation are pyruvate dehydrogenase E1 component subunit $\alpha$ (PDHE1 $\alpha$ ) of the pyruvate deydrogenase complex, long chain acyl- COA dehydrogenase (LCAD) of the $\beta$ oxidation machinery, ATP synthase $\alpha$ of the respiratory complex $\mathrm{V}$, translocase of outer membrane $70 \mathrm{kDa}$ subunit (TOM 70), voltage dependant anion channel 1 (VDAC1), creatine kinase S type and aconitate hydratase among others. All these proteins are members of important functional networks implicated at various times in heart failure and other cardiac diseases. This data renders it easy to focus further study on the particular proteins that have been modified by phosphorylation.

To have a reference data set to which the change in phosphoproteome can be compared, one study has evaluated the unstressed healthy murine models to elucidate 236 phosphosites belonging to 181 phospho proteins which include proteins of all major mitochondrial pathways ${ }^{[28]}$.

\section{Cardiac myofilament subproteome}

The cardiac myofilament has also been a subject of interest as it is the major contractile apparatus of the cell. Myofil- 
ament proteins like myosin, actin, titin are implicated in heart failure. The myofilament phosphoproteome was studied in a rat heart model treated with endothelin 1 and isoproterenol; potent inducers of myofilament phosphorylation. The study found an excess of 600 proteins to be phosphorylated other than several known proteins in the myofilament sub proteome. In particular, Isoproterenol treatment showed a shift in the subcellular localization of protein phospahatase $2 \mathrm{~A}$ regulatory subunit $\mathrm{B} 56 \alpha^{[29]}$. In another study the myofilament sub proteome was studied in case of acute MI in a swine model to reveal that there was a significant reduction in phosphorylation of important myofilament proteins like cardiac troponin I, myosin regulatory light chain and enigma homolog isoform $2^{[30]}$.

\section{Redox proteomics}

The redox status of the cell dictates the health and optimal functioning of proteins in the cell. An imbalance in this redox status leads to the formation of harmful free radicals and reactive oxygen species that have been decisively established as major causative factors behind cardiac diseases like myocardial infarction, ischemia-reperfusion injury, coronary heart disease, heart failure and even hypertrophy. Thus proteomic approaches have targeted the redox state of proteins to find out the global scenario during such cardiac diseases. A study has found that protein carbonylation, a result of oxidative stress, is increased in the myocardium during heart failure with M-type creatine kinase (M-CK) being the most affected. $\alpha$ cardiac actin is also affected to a lesser degree. They attempted to explain the defects in energy metabolism during heart failure based on their findings of loss of function of M-CK due to its increased carbonylation ${ }^{[31]}$. In case of ischemia-reperfusion, a study has found evident oxidative stress by increased accumulation of protein carbonyl. They found that thiol oxidation occurred mainly during ischemia or early reperfusion. Longer reperfusion led to reversal in thiol oxidation. Proteins involved in important heart functions like the proteins of the electron transport chain complexes- I, II, III, ATP synthase, long chain acetyl COA dehydrogenase and sarco/ endoplasmic reticulum $\mathrm{Ca}^{2+}$ ATPase (SERCA) showed oxidation of their thiol residues. It was concluded from the study that most oxidative changes were reversible up to certain duration in ischemia-reperfusion. However, increased protein carbonyl formation could be deleterious to the ischemic heart subjected to reperfusion ${ }^{[32]}$.

A lot of well known anti cancer drugs like Doxorubicin and Adriamycin are cardiotoxic. Redox effects of such drugs on the heart have also been studied by the redox proteomic approach. A Doxorubicin based study has found that it causes increased accumulation of 4-hydroxynonenal (HNE); a lipid peroxidation product in murine hearts. The proteins mostly affected belong to mitochondrial energy metabolism network ${ }^{[33]}$. Another Adriamycin based study found that specific carbonyl levels of three proteins- triose phosphate isomerise, $\beta$ enolase and electron transfer flavoprotein- ubiquinone oxidoreductase (ETF-QO) were significantly increased. They concluded that the mitochondrial bioenergetic pathway was a prime oxidative stress target of adriamycin in the heart ${ }^{[34]}$.

\section{Comparative proteomics}

This is a proteomic approach where two or more cardiac disease forms, two or more treatment procedures, temporal and spatial cardiac variations have been compared to delineate their similarities and differences and aid in development of better therapeutic strategies. Mitra et al. (2015) $)^{[7]}$ previously conducted a comparative proteomic study on rodent hearts suffering from two critical cardiac diseases- hypertrophy and myocardial infarction. It was found that altered glucose oxidation pattern distinguished the cellular energy levels and the disease types for hypertrophy and myocardial infarction. These investigators have also been able to elucidate in detail the differential apoptotic cascades that play an important role during cardiac deterioration process in etiologically different cardiac disease forms, i.e predominant mitochondrial and endoplasmic reticulum stress mediated myocyte death during hypertrophy and myocardial infarction respectively. A candidate protein found in this study was $\alpha$ Crystallin B which was shown to play a critical protective role during myocardial infarction by targeting to mitochondrial channel protein VDAC1 and blocking mitochondrial pathway of apoptosis. Further, the group has also shown that the cardioprotective effect of $\alpha$ Crystallin B is mediated by p38 MAPK during myocardial infarction ${ }^{[7,21,35]}$. Another comparative study between human ischemic and dilated cardiomyopathy revealed eleven proteins with similar expression patterns apart from the differentially expressed proteins. These eleven proteins could signify a common course of events that lead to heart failure by ischemic or dilated cardiomyopathy ${ }^{[36]}$.

Several other studies have been carried out using innovative proteomic approaches to micro dissect the cellular and molecular processes and events occurring in the heart during different diseases for better understanding and development of therapeutic care. Inclusion of all of them is beyond the scope of this review. As huge amounts of proteomic data is being churned out regularly by a large number of studies, keeping track of them is proving to be a difficult task. In order to simplify the storage and access to such data and reduce redundancy of research efforts a consolidated cardiac proteome knowledgebase has been developed. It has been named Cardiac Organellar Protein Atlas Knowledgebase (COPaKB). It is a centralised platform having cardiac proteomic data, bioinformatic tools for processing the data and relevant cardiovascular phenotypes ${ }^{[37]}$. Another project called the Cardiac Atlas Project (CAP) has also been established as a large scale database of cardiac images from clinical examinations along with associated clinical data. It has been set up as an atlas of the structural and functional aspects of normal and pathological heart ${ }^{[38]}$.

\section{Computational modelling in cardiac biology}

Modelling and simulation has been used in cardiac biology for a long time to help interpret a wide range of experimental data and integrate them into a highly detailed/ intricate visual system to give us a most comprehensive virtual organ ${ }^{[39]}$. The advancement in both non invasive cardiac imaging systems and computational methods has made it possible to design and use simulations and cardiac models that has enhanced our understanding of cardiac function ${ }^{[40,41]}$. This has made such model systems of infinite use in clinical settings in decision making and therapeutic intervention for various cardiac diseases. A comprehensive review on this topic has been made by Trayanova ${ }^{[39]}$.

Electrophysiological data from numerous experiments and biological model systems have been incorporated to give us the electromechanical models of the heart ${ }^{[39,42]}$. These mod- 
els integrate the electrical wave propagation in the heart and the mechanical contraction-relaxation performed by the contractile proteins of the myocytes that lead to the actual pumping of the ventricles ${ }^{[39]}$. The sequence of events that lead to ventricular contraction, namely, depolarization of myocytes by action potential, release of $\mathrm{Ca}^{2+}$ from intracellular stores and its binding to Troponin C, contraction of myocytes and ultimately ventricular deformation are all recorded to give a working electromechanical model of the heart. For the simulation of a cardiac cycle and corresponding pressure-volume loop conditions on chamber, volume and pressure are imposed by circulatory systems lumped-parameter models ${ }^{[39,40,42]}$. These models are utilised to answer for problems relating to the mechanical and electrophysiology of the heart in a generalised manner or in user specified individual cases [the geometry and structure as specified by user's magnetic resonance images (MRI)].

Whole heart models in cardiac electrophysiology have been extensively used to study normal wave propagation in healthy and diseased hearts and in cardiac arrhythmias ${ }^{[39]}$. In case of arrhythmias whole heart models reveal mechanism of causation and suggest methods of better therapy. Exploring arrhythmia was the first application of ventricular modelling and a number of ventricular models (3D models) have focused on arrhythmia dynamics ${ }^{[43-45]}$. They have been extensively used to characterise ventricular fibrillation ${ }^{[4]}$. Ventricular arrhythmia due to myocardial infarction has also been well characterised by such models ${ }^{[44,45]}$. In addition to ventricular models, MRI images and electrophysiological data for the atria has resulted in the construction of models to address atrial arrhythmias ${ }^{[45,46]}$. Electromechanical models have been used to design treatments for ventricular pump dysfunction with special uses in cardiac resynchronisation therapy (CRT). They have elucidated mechanisms that govern CRT efficacy ${ }^{[39,47]}$.

In patients with cardiovascular disease, assessing the risk of lethal arrhythmia and stratifying at-risk patients accordingly decreases mortality and health care costs. This has been considerably enhanced by using ventricular models along with electrocardiogram (ECG) data from patients ${ }^{[39]}$. In administering therapeutic interventions (surgical or otherwise) ventricular models are being used to make critical decisions ${ }^{[48]}$.

A new and challenging aspect in the realm of cardiac modelling is the application of such models for individual patients customizing for their particular cardiac problems. Enhanced imaging systems notwithstanding several obstacles in this process are the acquisition of poor in vivo scans, the perpetually loaded state of the heart during image acquisition that provide difficulty in modelling the geometric mesh of the patient's heart ${ }^{[36]}$. However, much progress has been made to overcome such problems and make individual modelling a prevailing procedure for cardiac research and therapy. New 3D models that incorporate patient specific data to give very faithful representations are being produced ${ }^{[42]}$. Computational cardiac modelling is thus merging the experimental data with complex programming to give a rich virtual visual representation of heart function.

\section{Conclusion}

Computational modelling on the one hand and proteomics on the other are two invaluable systems biology approaches that are being increasingly employed in cardiac research. Car- diac models and simulations are enhancing the interpretation of experimental data and systematic storage of vast amounts of proteomics data is being achieved by developing comprehensive knowledge bases. Proteomics is by far the most relevant systems biology method that is being applied in every area of cardiac research to provide global as well as definite information to answer pressing concerns of cardiac diseases.

\section{References}

1. Cardiovascular Diseases (CVDs). Fact sheet Nº317. (2015) World Health Organization.

2. Hyung, S.J., Ruotolo, B.T. Integrating mass spectrometry of intact protein complexes into structural proteomics. (2012) Proteomics 12(10): 1547-1564.

3. Yang, L., Chen, J., Shi, L., et al. Identifying unexpected therapeutic targets via chemical-protein interactome. (2010) PLoS One 5(3): e9568. 4. Yang, L., Wang, K., Chen, J., et al. Exploring off-targets and off-systems for adverse drug reactions via chemical-protein interactome--clozapine-induced agranulocytosis as a case study. (2011) PLoS Comput Biol 7(3): e1002016.

5. Sakai, J., Ishikawa, H., Satoh, H., et al. Two dimensional differential gel electrophoresis of rat heart proteins in ischemia and ischemia-reperfusion. (2007) Methods Mol Biol 357: 33-43.

6. Haas, S., Jahnke, H.G., Moerbt, N., et al. DIGE proteome analysis reveals suitability of ischemic cardiac in vitro model for studying cellular response to acute ischemia and regeneration. (2012) PLoS One 7(2): e31669.

7. Mitra, A., Basak, T., Ahmad, S., et al. Comparative Proteome Profiling during Cardiac Hypertrophy and Myocardial Infarction Reveals Altered Glucose Oxidation by Differential Activation of Pyruvate Dehydrogenase E1 Component Subunit $\beta$. (2015) J Mol Biol 427(11): 2104-2120.

8. Jüllig, M., Hickey, A.J., Middleditch, M.J., et al. Characterization of proteomic changes in cardiac mitochondria in streptozotocin-diabetic rats using iTRAQ ${ }^{\mathrm{TM}}$ isobaric tags. (2007) Proteomics Clin Appl 1(6): 565-576.

9. Warren, C.M., Geenen, D.L., Helseth, D.L., et al. Sub-proteomic fractionation, iTRAQ, and OFFGEL-LC-MS/MS approaches to cardiac proteomics. (2010) J Proteomics 73(8):1551-1561.

10. Rocchiccioli, J.P., McMurray, J.J., Dominiczak, A.F. Biomarkers in heart failure: a clinical review. (2010) Heart Fail Rev 15(4): 251-273.

11. Hochholzer, W., Morrow, D.A., Giugliano, R.P. Novel biomarkers in cardiovascular disease: Update 2010. (2010) Am Heart J 160(4): 583-594.

12. Ridker, P.M. C-Reactive Protein: Eighty Years from Discovery to Emergence as a Major Risk Marker for Cardiovascular Disease. (2009) Clin Chem 55(2): 209-215.

13. Jacquet, S., Yin, X., Sicard, P., et al. Identification of cardiac myosin-binding protein $\mathrm{C}$ as a candidate biomarker of myocardial infarction by proteomics analysis. (2009) Mol Cell Proteomics 8(12): 2687-2699. 14. Haas, B., Serchi, T., Wagner, D.R., et al. Proteomic analysis of plasma samples from patients with acute myocardial infarction identifies haptoglobin as a potential prognostic biomarker. (2011) J Proteomics 75(1): 229-236.

15. Mebazaa, A., Vanpoucke, G., Thomas, G., et al. Unbiased plasma proteomics for novel diagnostic biomarkers in cardiovascular disease: identification of quiescin Q6 as a candidate biomarker of acutely decompensated heart failure. (2012) Eur Heart J 33(18): 2317-2324.

16. Jiang, H., Zhang, L., Yu, Y., et al. A pilot study of angiogenin in heart failure with preserved ejection fraction: a novel potential biomarker for diagnosis and prognosis? (2014) J Cell Mol Med 18(11): 2189-2197.

17. Kakimoto, Y., Ito, S., Abiru, H., et al. Sorbin and SH3 domain-containing protein 2 is released from infarcted heart in the very early phase: proteomic analysis of cardiac tissues from patients. (2013) J Am Heart Assoc 2(6): e000565. 
18. Li, W., Rong, R., Zhao, S., et al. Proteomic analysis of metabolic, cytoskeletal and stress response proteins in human heart failure. (2012) J Cell Mol Med 16(1):59-71.

19. Urbonavicius, S., Wiggers, H., Bøtker, H.E., et al. Proteomic analysis identifies mitochondrial metabolic enzymes as major discriminators between different stages of the failing human myocardium. (2009) Acta Cardiol 64(4): 511-522.

20. Schechter, M.A., Hsieh, M.K., Njoroge, L.W., et al. Phosphoproteomic profiling of human myocardial tissues distinguishes ischemic from non-ischemic end stage heart failure. (2014) PLoS One 9(8): e104157.

21. Mitra, A., Basak, T., Datta, K., et al. Role of $\alpha$-crystallin B as a regulatory switch in modulating cardiomyocyte apoptosis by mitochondria or endoplasmic reticulum during cardiac hypertrophy and myocardial infarction. (2013) Cell Death Dis 4: e582.

22. Agnetti, G., Husberg, C., Van Eyk, J.E. Divide and conquer: the application of organelle proteomics to heart failure. (2011) Circ Res 108(4): 512-526.

23. Wang, Y., Li, C., Chuo, W., et al. Integrated proteomic and metabolomic analysis reveals the NADH-mediated TCA cycle and energy metabolism disorders based on a new model of chronic progressive heart failure. (2013) Mol Biosyst 9(12): 3135-3145.

24. Li, X., Arslan, F., Ren, Y., et al. Metabolic adaptation to a disruption in oxygen supply during myocardial ischemia and reperfusion is underpinned by temporal and quantitative changes in the cardiac proteome. (2012) J Proteome Res 11(4): 2331-2346.

25. Giorgianni, F., Koirala, D., Weber, K.T., et al. Proteome analysis of subsarcolemmal cardiomyocyte mitochondria: a comparison of different analytical platforms. (2014) Int J Mol Sci 15(6): 9285-9301.

26. Giorgianni, F., Usman Khan, M., Weber, K.T., et al. Phosphoproteome mapping of cardiomyocyte mitochondria in a rat model of heart failure. (2014) Mol Cell Biochem 389(1-2): 159-167.

27. Zhao, X., Leon, I.R., Bak, S., et al. Phosphoproteome analysis of functional mitochondria isolated from resting human muscle reveals extensive phosphorylation of inner membrane protein complexes and enzymes. (2011) Mol Cell Proteomics 10(1): M110.000299.

28. Deng, N., Zhang, J., Zong, C., Phosphoproteome analysis reveals regulatory sites in major pathways of cardiac mitochondria. (2011) Mol Cell Proteomics 10(2): M110.000117.

29. Yin, X., Cuello, F., Mayr, U., et al. Proteomics analysis of the cardiac myofilament subproteome reveals dynamic alterations in phosphatase subunit distribution. (2010) Mol Cell Proteomics 9(3): 497-509.

30. Peng, Y., Gregorich, Z.R., Valeja, S.G., et al. Top-down proteomics reveals concerted reductions in myofilament and Z-disc protein phosphorylation after acute myocardial infarction. (2014) Mol Cell Proteomics 13(10): 2752-2764.

31. Brioschi, M., Polvani, G., Fratto, P., et al. Redox proteomics identification of oxidatively modified myocardial proteins in human heart failure: implications for protein function. (2012) PLoS One 7(5): e35841. 32. Kumar, V., Kleffmann, T., Hampton, M.B., et al. Redox proteomics of thiol proteins in mouse heart during ischemia/reperfusion using ICAT reagents and mass spectrometry. (2013) Free Radic Biol Med 58: 109-117.
33. Zhao, Y., Miriyala, S., Miao, L., et al. Redox proteomic identification of HNE-bound mitochondrial proteins in cardiac tissues reveals a systemic effect on energy metabolism after doxorubicin treatment. (2014) Free Radic Biol Med 72: 55-65.

34. Chen, Y., Daosukho, C., Opii, W.O., et al. Redox proteomic identification of oxidized cardiac proteins in adriamycin-treated mice. (2006) Free Radic Biol Med 41(9): 1470-1477.

35. Mitra, A., Ray, A., Datta, R., et al. Cardioprotective role of P38 MAPK during myocardial infarction via parallel activation of $\alpha$-crystallin B and Nrf2. (2014) J Cell Physiol 229(9): 1272-1282.

36. Roselló-Lletí, E., Alonso, J., Cortés, R., et al. Cardiac protein changes in ischaemic and dilated cardiomyopathy: a proteomic study of human left ventricular tissue. (2012) J Cell Mol Med 16(10): 2471-2486. 37. Zong, N.C., Li, H., Li, H., et al. Integration of cardiac proteome biology and medicine by a specialized knowledgebase. (2013) Circ Res 113(9): 1043-1053.

38. Fonseca, C.G., Backhaus, M., Bluemke, D.A., et al. The Cardiac Atlas Project--an imaging database for computational modeling and statistical atlases of the heart. (2011) Bioinformatics 27(16): 2288-2295.

39. Trayanova, N.A. Whole-heart modeling: applications to cardiac electrophysiology and electromechanics. (2011) Circ Res 108(1): 113128.

40. Gurev, V., Lee, T., Constantino, J., et al. Models of cardiac electromechanics based on individual hearts imaging data: Image-based electromechanical models of the heart. (2011) Biomech Model Mechanobiol 10(3): 295-306.

41. Deng, D., Jiao, P., Ye, X.,et al. An image-based model of the whole human heart with detailed anatomical structure and fiber orientation. (2012) Comput Math Methods Med 2012: 891070.

42. Sermesant, M., Delingette, H., Ayache, N. An electromechanical model of the heart for image analysis and simulation. (2006) IEEE Trans Med Imaging 25(5): 612-625.

43. Trayanova, N.A., Constantino, J., Gurev, V. Models of stretch-activated ventricular arrhythmias. (2010) J Electrocardiol 43(6): 479-485. 44. Yamaki, M., Kubota, I., Tomoike, H. Simulation of late potentials and arrhythmias by use of a three-dimensional heart model: casuality of peri-infarctional slow conduction in ventricular fibrillation. (1999) J Electrocardiol 32(2): 115-121.

45. Lopez-Perez, A., Sebastian, R., Ferrero, J.M. Three-dimensional cardiac computational modelling: methods, features and applications. (2015) Biomed Eng Online 14: 35.

46. Tobón, C., Ruiz-Villa, C.A., Heidenreich, E., et al. A three-dimensional human atrial model with fiber orientation. Electrograms and arrhythmic activation patterns relationship. (2013) PLoS One 8(2): e50883.

47. Reumann, M., Farina, D., Miri, R., et al. Computer model for the optimization of AV and VV delay in cardiac resynchronization therapy. (2007) Med Biol Eng Comput 45(9): 845-854.

48. Lee, L.C., Genet, M., Dang, A.B., et al. Applications of computational modeling in cardiac surgery. (2014) J Card Surg 29(3): 293-302.
Ommega Online Publisher

Bioinformatics, Proteomics and Immaging Analysis

Short Title : Bioinfo Proteom Img Anal
ISSN: 2381-0793

E-mail : bioinfo@ommegaonline.org

website: www.ommegaonline.org 